\title{
Chloronychia in Healthcare Workers in COVID-19 Times
}

\author{
Anca E. Chiriac ${ }^{a}$ Anca Chiriac $^{b, c, d}$ Uwe Wollina $^{e}$ \\ aEpidemiology Department, "Grigore T. Popa" University of Medicine and Pharmacy, lasi, Romania; \\ ${ }^{b}$ Department of Dermatology, Nicolina Medical Center, lasi, Romania; ' Department of Dermatology, Apollonia \\ University, lasi, Romania; ' "P. Poni” Institute of Macromolecular Chemistry, Romanian Academy, Iasi, Romania; \\ e Department of Dermatology and Allergology, Städtisches Klinikum Dresden, Academic Teaching Hospital of \\ the Technical University of Dresden, Dresden, Germany
}

\section{Dear Editor,}

A recent study published that $80 \%$ of 61 patients hospitalized in the intensive care unit for COVID-19 acute respiratory infection had bronchial aspirates positive for Pseudomonas aeruginosa [1]. On the other hand, no $P$. aeruginosa infection has been reported affecting the skin of patients or of healthcare providers.

It is well known today that variable skin disorders are induced by protective equipment such as facemasks and gloves. Prolonged use of gloves can cause hyperhidrosis, moisture, and humid areas, which can favor colonization of $P$. aeruginosa. $P$. aeruginosa is named "the water bug" because it does survive only in humid environments.

$P$. aeruginosa is a Gram-negative aerobic coccobacillus, which is not part of the normal skin flora and, in immunocompetent individuals, does not cause any skin infection. In immunosuppressed patients, $P$. aeruginosa can be the pathogen causing pneumonia, osteomyelitis, and even sepsis [2].

$P$. aeruginosa nail infection is quite characteristic due to blue-green discoloration of the nail plate caused by two pigments: pyocyanin and pyoverdin (Fig. 1).

Chloronychia observed in healthcare workers can be explained by the humid environment and possible associated nail lesions, but could it be a risk factor for immuno-

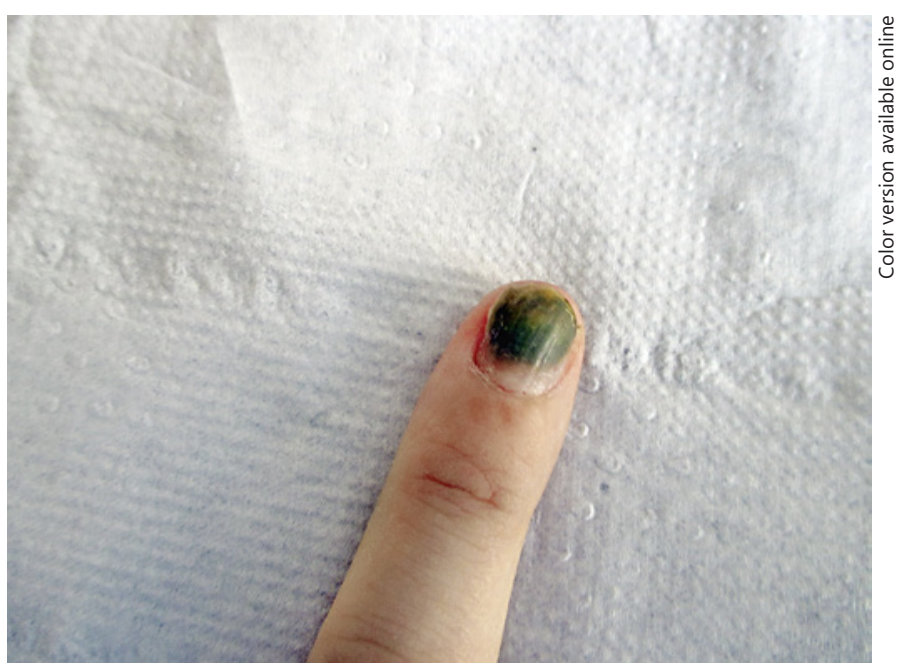

Fig. 1. Blue-green discoloration of the nail plate (the nail has become onycholytic, according to the patient's history, only after the color change had been noted).

suppressed patients? $P$. aeruginosa can easily spread due to scratching and cause soft tissue infections such as ecthyma gangrenosum or necrotizing gangrene in immunocompromized individuals. karger@karger.com www.karger.com/sad

(C) 2020 S. Karger AG, Basel

Karger'
Anca Chiriac

Department of Dermatology, Nicolina Medical Center

Hatman Sendrea Street, 2

RO-700613 Iasi (Romania)

ancachiriac@yahoo.com 
$P$. aeruginosa nail infection could be an occupational skin disease among healthcare providers, but it may also be a concern by spreading the infection to others, especially to hospitalized patients in COVID-19 units.

\section{Conflict of Interest Statement}

The authors have no conflicts of interest to declare.

\section{Funding Sources}

The authors did not receive any funding.

\section{Author Contributions}

All authors equally contributed to this work.

References

1 Chen J, Zheng YX, Kong DC, Wu HY, Yuan ZA, Wu F. [Practice and thinking of acute respiratory infection surveillance for the response of emerging respiratory diseases in Shanghai]. Zhonghua Liu Xing Bing Xue Za Zhi. 2020 Jun;41(0):E073.

2 Schwartz RA, Reynoso-Vasquez N, Kapila R. Chloronychia: The Goldman-Fox Syndrome - Implications for Patients and Healthcare Workers. Indian J Dermatol. 2020 Jan-Feb; 65(1):1-4. 